2020, Instituto Mexicano de Tecnología del Agua

Tecnología y

Ciencias $\stackrel{\unlhd}{\unlhd}$ gua
Open Access bajo la licencia CC BY-NC-SA 4.0 (https://creativecommons.org/licenses/by-nc$\mathrm{sa} / 4.0 /)$

DOI: $10.24850 / j-$ tyca-2020-06-08

Artículos

\title{
Prediciendo la dosis de sulfato de aluminio en el tratamiento de aguas
}

\section{Predicting the aluminum sulfate dosage in water treatment}

Ana María Morales ${ }^{1}$, ORCID: https://orcid.org/0000-0002-0103-1277

Gustavo Ramírez-Caballero², ORCID: https://orcid.org/0000-0001-96654022

Martha Barajas-Meneses33, ORCID: https://orcid.org/0000-0001-69549526

${ }^{1}$ Grupo de Investigación en Polímeros, Escuela de Ingeniería Química, Universidad Industrial de Santander, Bucaramanga, Colombia, mariaana91_@hotmail.com

${ }^{2}$ Grupo de Investigación en Polímeros, Escuela de Ingeniería Química, Universidad Industrial de Santander, Bucaramanga, Colombia, gusramca@uis.edu.co

${ }^{3}$ Grupo de Investigación en Polímeros, Escuela de Ingeniería Química, Universidad Industrial de Santander, Bucaramanga, Colombia, marthabm@uis.edu.co 


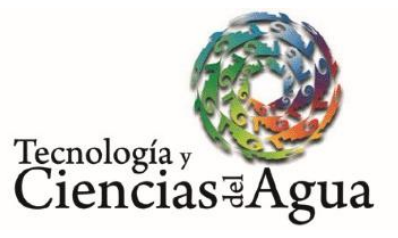

2020, Instituto Mexicano de Tecnología del Agua Open Access bajo la licencia CC BY-NC-SA 4.0 (https://creativecommons.org/licenses/by-nc$\mathrm{sa} / 4.0 /$ )

Autora para correspondencia: Martha Barajas-Meneses, marthabm@uis.edu.co

\section{Resumen}

El presente estudio muestra las estrategias usadas para mejorar el proceso de clarificación en la planta de agua desmineralizada en la planta GENSA S. A. E. S. P., de Termopaipa, localizada en Boyacá, Colombia. Se emplearon datos experimentales obtenidos a partir de la prueba de jarras para construir un modelo basado en redes neuronales. Las variables independientes fueron $\mathrm{pH}$, turbiedad, conductividad eléctrica y color del agua cruda, junto con la dosis de polímero floculante. La variable de salida del modelo fue la dosis del coagulante. Se escogió el modelo de una red neuronal de tres capas, el cual fue validado para encontrar 10 neuronas en la capa oculta. La herramienta para entrenar la red neuronal fue optimización no lineal. El cálculo de chi cuadrada utilizado para la evaluación del modelo demostró ser eficiente en un $90 \%$ de nivel de confianza.

Palabras clave: coagulación, correlación, tratamiento de agua, redes neuronales.

\section{Abstract}

The present study shows the strategies used to improve the treatment of clarification of demineralized water in GENSA S. A. E. S. P., Planta Termopaipa, located in Boyacá, Colombia. Experimental data obtained from jar tests were used to build a model based on neuronal nets. The independent variables were $\mathrm{pH}$, turbidity, electrical conductivity, and color 


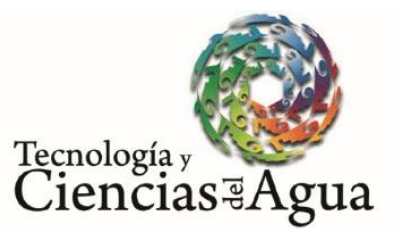
2020, Instituto Mexicano de Tecnología del Agua Open Access bajo la licencia CC BY-NC-SA 4.0 (https://creativecommons.org/licenses/by-nc- $\mathrm{sa} / 4.0 /$ )

of the raw water along with flocculent dosage. The output variable was the Aluminum Sulfate dosage. A three-layer neural network was chosen as a prediction approach. The model was validated to find ten neurons in the hidden layer. Nonlinear optimization was the tool used to train the neural network. The chi-square value was used to test the model and showed that the model is efficient at $90 \%$ confidence level.

Keywords: Coagulation, correlation, cross-validation, neural net, water treatment.

Recibido: 15/06/2019

Aceptado: $10 / 04 / 2020$

\section{Introducción}

El tratamiento del agua para sistemas de enfriamiento y calderas es fundamental para cualquier planta termoeléctrica e incluye el tratamiento convencional y de desionización. El tratamiento convencional del agua se compone de las etapas de coagulación, floculación, sedimentación y filtración, que conducen a la mayor remoción de turbiedad. El proceso coagulación-floculación es un pretratamiento fisicoquímico que requiere de sustancias químicas, las cuales permiten que las partículas se 


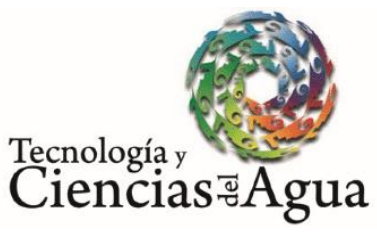

2020, Instituto Mexicano de Tecnología del Agua Open Access bajo la licencia CC BY-NC-SA 4.0 (https://creativecommons.org/licenses/by-nc$\mathrm{sa} / 4.0 /)$

aglomeren. El tratamiento de desionización conlleva la reducción de iones disueltos y se realiza a través de materiales con capacidad de intercambio iónico (Bhatnagar \& Minocha, 2006) y materiales con capacidad de adsorción (Machado-Infante, Ramírez-Caballero, \& Barajas-Meneses, 2016). Con el fin de disminuir los costos en el tratamiento del agua, una de las estrategias es la optimización de las cantidades de los materiales requeridos, así que uno de los primeros pasos será la optimización de la dosis de coagulante en el tratamiento convencional.

La prueba de jarras es reconocida en la industria como una herramienta valiosa para simular y optimizar el proceso de coagulación, floculación y sedimentación, lo que permite hacer experimentos, evitando manipular la planta de tratamiento directamente. Un modelo matemático empleando los datos experimentales obtenidos a través de prueba de jarras puede implementarse para predecir la eficiencia de coagulantes en el tratamiento convencional. La utilización de un modelo evita efectuar experimentos adicionales de prueba de jarras que consumen tiempo y materiales. Uno de los modelos más comunes son las redes neuronales artificiales, los cuales son nodos que, como las neuronas del cerebro, están conectadas entre sí y reciben, procesan y transmiten señales provenientes de estímulos externos. Las redes neuronales, como el cerebro, aprenden de la experiencia para cumplir funciones cognitivas como la predicción. Las variables predichas por las redes neuronales son resultado de funciones no lineales de las variables externas. Por ejemplo, los datos de porcentaje de remoción de turbiedad obtenidos con la semilla de mango, la cual se usó como coagulante durante la prueba de jarras, se aplicaron en un modelo basado en una red neuronal de tres capas. El modelo empleó como variables de entrada turbiedad, $\mathrm{pH}$, tiempo de 


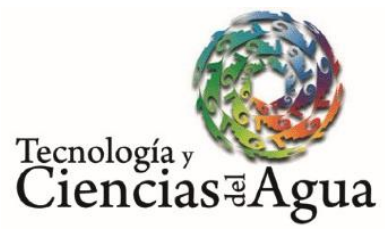

2020, Instituto Mexicano de Tecnología del Agua Open Access bajo la licencia CC BY-NC-SA 4.0 (https://creativecommons.org/licenses/by-ncsa/4.0/)

sedimentación, dosis de semilla de mango y tiempo de contacto. Los valores de coeficientes de determinación $R^{2}$ muestran cercanía entre los valores predichos por el modelo y los valores experimentales (Olusola, Olurotimi, Adekilekun, \& Adetayo, 2019). En otro caso se utilizaron modelos de redes neuronales para predecir la turbiedad removida y se usaron como variables de entrada turbiedad, alcalinidad, dureza, $\mathrm{pH}$, temperatura del agua cruda, dosis de alumbre, dosis de permanganato y dosis de dióxido de cloro. Las redes neuronales incluyeron aquellas de perceptrón de multicapa donde los pesos se inicializaron por algoritmos genéticos y también por recocido simulado; del mismo modo, mostraron ser capaces de predecir la turbiedad removida con altos coeficientes de correlación (Kennedy, Gandomi, \& Miller, 2015).

En general, los modelos basados en redes neuronales son ampliamente usados para cuantificar los efectos de algunos adsorbentes para el tratamiento de aguas. Por ejemplo, una red de tres capas permitió predecir el porcentaje de remoción de microcontaminantes cuando un composite basado en quitosano magnético y biocarbón se emplea como adsorbente en un sistema similar al de la prueba de jarras. Los valores de entrada al modelo fueron la concentración de microcontaminantes y el $\mathrm{pH}$. Los coeficientes de determinación $R^{2}$ para evaluar la eficacia de la predicción de la red neuronal indicaron una buena proximidad entre los valores predichos por el modelo y los valores experimentales correspondientes al porcentaje de remoción de microcontaminantes (Mojiri, Kazeroon, \& Gholami, 2019). En otro estudio se utilizó biomasa crecida del Bacillus thuringiensis strain W3 para adsorber As (III). La eficiencia de adsorción se modeló en una red neuronal de tres capas. La primera, capa de variables de entrada; la segunda, una capa oculta, y la 


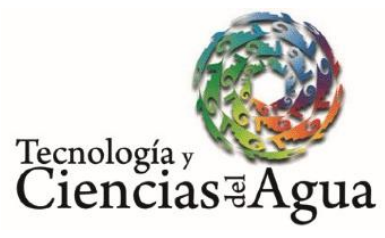
2020, Instituto Mexicano de Tecnología del Agua Open Access bajo la licencia CC BY-NC-SA 4.0 (https://creativecommons.org/licenses/by-nc- $\mathrm{sa} / 4.0 /$ )

tercera, la variable de salida. Las variables de entrada consistieron en la concentración de As (III), dosis del bioadsorbente, temperatura, $\mathrm{pH}$ y tiempo. Los coeficientes de determinación $R^{2}$ mostraron una efectiva simulación para predecir la adsorción de los iones de arsénico (Altowayti, Algaifi, Bakar, \& Shahir, 2019).

Termopaipa, Gensa S.A. E.S.P. es una de las termoeléctricas más grandes en Colombia, con capacidad de producción de 328 MW. De agosto a diciembre de 2014 se llevaron a cabo una serie de experimentos de pruebas de jarras para determinar las dosis óptimas de sulfato de aluminio como coagulante para reducir la turbiedad del agua cruda al mínimo posible. Con el objetivo de poder ejecutarpredicciones de la dosis óptima y así evitar experimentos adicionales con la prueba de jarras, se implementó una red unidireccional. Se utilizaron los coeficientes de correlación de Pearson para estudiar la relación entre variables. Del mismo modo, se empleó la optimización no lineal como herramienta para entrenar la red neuronal, usando Microsoft Excel Solver. La validación cruzada se hizo para obtener la mejor arquitectura de la red neuronal. La eficiencia de la red neuronal se demostró a través de los coeficientes de chi cuadrada.

\section{Materiales y métodos}




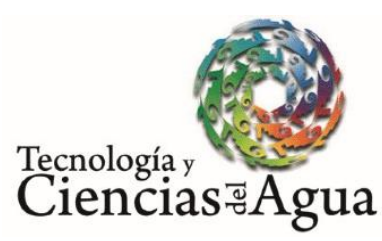

2020, Instituto Mexicano de Tecnología del Agua Open Access bajo la licencia CC BY-NC-SA 4.0 (https://creativecommons.org/licenses/by-nc$\mathrm{sa} / 4.0 /$ )

\section{Prueba de jarras y parámetros de calidad del agua}

El agua usada para la prueba de jarras se obtuvo del mismo lago que alimenta la planta termoeléctrica. El coagulante elegido fue el sulfato de aluminio y el polímero floculante la poliacrilamida aniónica. El mezclado rápido de la coagulación se llevó a cabo a 100 rpm y reducido a $40 \mathrm{rpm}$ para el mezclado lento de la floculación.

El pH, la turbiedad, la conductividad eléctrica y el color se midieron en el agua que alimenta la planta en unidades de $\mathrm{pH}$, unidades nefelométricas de turbiedad (UNT), conductividad eléctrica (microsiemens/cm, $\mu \mathrm{S} / \mathrm{cm}$ ) y unidades de platino cobalto (UPC), respectivamente. El equipo utilizado para la prueba de jarras fue de seis jarras con agitadores mecánicos de paleta, de marca Phipps \& Bird. El pH se midió con un equipo WTW ProfiLine pH 3110; la turbiedad, con un nefelómetro WTW TurB 430 IR; la conductividad eléctrica, con un conductímetro Cond 3210; finalmente, el color se midió con un espectrofotómetro HACH DR 5000. Para el entrenamiento de la red neuronal se usó un total de 27 datos experimentales; cuatro de ellos (obtenidos durante la prueba jarras) se emplearon para evaluar el modelo.

\section{Asociación e independencia de las variables}


Tecnología y

Ciencias $₫$ Agua
2020, Instituto Mexicano de Tecnología del Agua Open Access bajo la licencia CC BY-NC-SA 4.0 (https://creativecommons.org/licenses/by-nc$\mathrm{sa} / 4.0 /$ )

Los coeficientes de correlación son útiles para detección extrema de colinearidad entre pares de variables, lo que conduciría a problemas como cambios grandes en la respuesta del modelo, cuando ocurren pequeños ajustes en las variables predictoras, haciendo que el modelo sea inestable. Los resultados interpretativos pueden ser erróneos si la colinearidad está presente en el modelo. Si éste es el caso, medidas como sólo seleccionar una, del par de variables altamente correlacionadas, permiten evitar tal clase de problemas (Mason \& Perreault, 1991).

Algunos autores recomiendan 0.9 , otros 0.7 , y otros aconsejan 0.5 como valores máximos de correlación para establecer cuando dos variables se correlacionan. La simplicidad de este método para inspeccionar la colinearidad en los datos experimentales para la construcción de modelos multivariables lo hace muy conveniente (Jayaweera \& Azis, 2018; Michael, Trinogga, y Cebrian-Piqueras, 2019):

$$
R_{x y}=\frac{\sum_{i=1}^{n}\left(x_{i}-\bar{x}\right)\left(y_{i}-\bar{y}\right)}{\sqrt{\sum_{i=1}^{n}\left(x_{i}-\bar{x}\right)^{2} \sum_{i=1}^{n}\left(y_{\mathrm{i}}-\bar{y}\right)^{2}}}
$$

\section{Arquitectura de la red neuronal y validación}




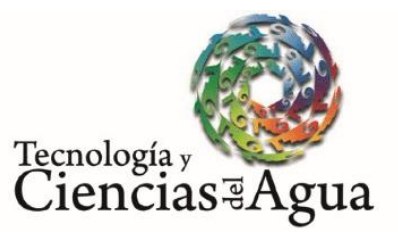
2020, Instituto Mexicano de Tecnología del Agua Open Access bajo la licencia CC BY-NC-SA 4.0 (https://creativecommons.org/licenses/by-nc- $\mathrm{sa} / 4.0 /$ )

La arquitectura de la red neuronal usada en este estudio es un perceptrón de multicapa y corresponde a la clase de red neuronal unidireccional, donde la información fluye desde la primera capa hasta la última sin permitir ciclos de retroalimentación.

Un ejemplo de red neuronal se representa en la Figura 1. La primera capa es una neurona pasiva; consiste en $D$ neuronas debido a la dimensionalidad $D$ de los datos experimentales. La segunda capa corresponde a la capa oculta que reciben los datos, los multiplica por el primer set de pesos, los combina de manera lineal y por último los alimenta a una función sigmoidea. La tercera capa representa la de salida, recibe la información de la segunda capa, los combina linealmente y alimenta una segunda función sigmoidea. Para este estudio se utilizó validación cruzada, a fin de calcular un número óptimo de neuronas en la capa oculta.

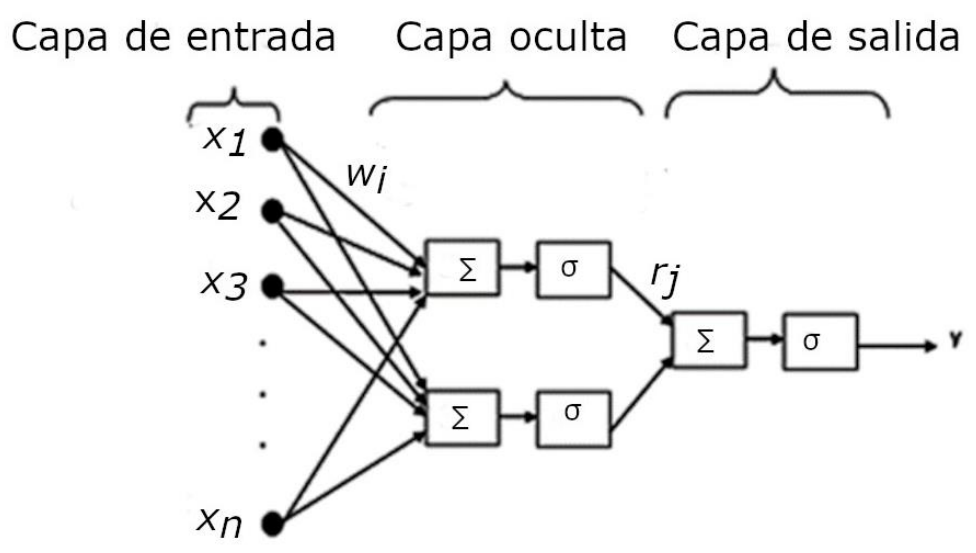

Figura 1. Ejemplo de una red neuronal que corresponde a un perceptrón multilineal de tres capas, con $D$ neuronas en la capa de entrada, dos neuronas en la capa oculta y una neurona en la capa de salida. 
2020, Instituto Mexicano de Tecnología del Agua

Tecnología y

Ciencias $\stackrel{\Xi}{\Im}$ Agua
Open Access bajo la licencia CC BY-NC-SA 4.0 (https://creativecommons.org/licenses/by-ncsa/4.0/)

La variable de salida para la red neuronal se representa así:

$y=\sigma_{2}\left[\sum_{j=1}^{H}\left(r_{j} \sigma_{1}\left(\sum_{i=1}^{D} w_{i} x_{i}\right)\right)\right]$

Donde $H$ es el número de neuronas en la capa oculta; $D$, la dimensionalidad de los datos experimentales que alimentan el modelo; $w_{i}$, el primer set de pesos sinápticos; $r_{j}$, el segundo set de pesos sinápticos y el valor predicho; $\sigma_{1}$, la primera función sigmoidea, y $\sigma_{2}$ es la segunda.

El entrenamiento de la red neuronal consiste en encontrar los pesos sinápticos a través de todo el espacio que minimiza el error entre los valores predichos por el modelo y los valores de la variable de salida de los datos experimentales. La búsqueda de tales pesos sinápticos óptimos empieza con valores iniciales y se van ajustando, agregando un valor de $\Delta w_{i}:$

$w_{1+i}=\mathrm{w}_{\mathrm{i}}+\Delta w_{i}$

$\Delta w_{i}=\gamma \mu_{k}$

Donde $y$ es la tasa de aprendizaje y $\mu k$ es la dirección de la búsqueda.

Para este estudio se utilizó el algoritmo de gradiente reducido generalizado, dado por Solver Excel, que consiste en la proyección del 
2020, Instituto Mexicano de Tecnología del Agua

Tecnología y

Ciencias $\stackrel{\Xi}{\unlhd}$ gua
Open Access bajo la licencia CC BY-NC-SA 4.0 (https://creativecommons.org/licenses/by-nc$\mathrm{sa} / 4.0 /)$

gradiente original en la región descrita por los pesos sinápticos. La dirección en que el gradiente reducido se desvanece permite encontrar la dirección de búsqueda del punto óptimo:

$\mu_{k}=-g_{R}$

Donde $g_{R}$ es el gradiente reducido generalizado (Rao, 2009).

Para validar el modelo y lograr un uso eficiente del número de datos experimentales, principalmente cuando éste no es tan alto, se utiliza la validación cruzada. En este tipo de validación, los datos para la evaluación del modelo se toman de los mismos datos experimentales de entrenamiento. Así, se pueden desarrollar varios modelos donde los datos se turnan entre los datos de entrenamiento y de evaluación para cada modelo; entonces, en lugar de desarrollar un solo modelo existen varios de un solo tipo, en los que también es posible evaluar su eficacia de predicción a través del error cuadrado predictivo (ECP).

En este trabajo, uno de los 27 datos experimentales para el entrenamiento de la red neuronal se excluyó, para emplearse en la evaluación del modelo, que resulta del entrenamiento con los otros 26 datos. Cada vez que se excluya un dato experimental diferente de los 27 que hay, es posible crear un modelo distinto; por lo tanto, se pueden desarrollar 27 modelos, cada uno con un dato de evaluación diferente. A cada modelo se le puede evaluar el ECP usando el dato excluido para la evaluación del modelo:

$E C P=\left(Y_{(-i)}-y_{(-i)}\right)^{2}$ 
Tecnología y

Ciencias $\stackrel{\Xi}{\unlhd}$ gua
2020, Instituto Mexicano de Tecnología del Agua Open Access bajo la licencia CC BY-NC-SA 4.0 (https://creativecommons.org/licenses/by-nc$\mathrm{sa} / 4.0 /$ )

ECP consiste en la diferencia al cuadrado del dato experimental $Y(-i)$, del dato excluido para la evaluación con respecto al $y(-i)$, que es predicho por el modelo. En el caso estudiado, los tipos de modelos corresponden a redes neuronales con diferentes arquitecturas. Por tanto, se construyen 27 modelos para cada red neuronal de diferente arquitectura con 2, 4, 6, 8, 10 y 12 neuronas en la capa oculta, para un total de 162 modelos. Para cada arquitectura o tipo de modelo es posible identificar el error de validación cruzada (EVC) de la siguiente forma:

$E V C=\frac{\sum_{i}^{27} E C P}{k}$

La arquitectura que tenga un menor valor de EVC será considerada la más óptima (Anders \& Korn, 1999; Hadzima-Nyarko, Nyarko, \& Ademovic, 2019).

\section{Evaluación del modelo}

Para la evaluación del modelo, el valor de chi cuadrada se calculó de esta manera: 
2020, Instituto Mexicano de Tecnología del Agua

Tecnología y

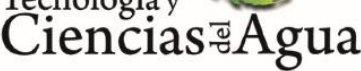
Open Access bajo la licencia CC BY-NC-SA 4.0 (https://creativecommons.org/licenses/by-nc$\mathrm{sa} / 4.0 /)$

$\chi^{2}=\sum_{i}^{N} \frac{\left(y_{i}-Y_{i}\right)^{2}}{y_{i}}$

Donde $Y_{i}$ es cada valor observado experimentalmente; $y_{i}$ corresponde a cada valor predicho, y $N$ es el número de total de datos experimentales usados en la evaluación del modelo. La comparación de este valor con un valor crítico dado por la distribución de chi cuadrada a cierto nivel de confianza, por lo general 95 o $90 \%$ para N-1 grados de libertad, rechaza o acepta la hipótesis nula de que $y_{i}$ y $Y_{i}$ no presentan diferencias significativas. Si el valor de chi cuadrada es menor que el valor crítico, entonces es posible aceptar la hipótesis nula, y concluir que no hay diferencias significativas entre los valores predichos y los experimentales. Por el contrario, si el valor chi cuadrada es mayor que el valor crítico, se puede rechazar la hipótesis nula y concluir que $Y_{i}$ y $y_{i}$ presentan diferencias significativas y por lo tanto el modelo no sería confiable. También se calcularon los residuales entre los datos experimentales y los datos predichos para los datos de entrenamiento.

\section{Resultados}

\section{Prueba de jarras y parámetros de calidad del agua}


Tecnología y

Ciencias $\stackrel{\unlhd}{\triangleleft}$ gua
2020, Instituto Mexicano de Tecnología del Agua Open Access bajo la licencia CC BY-NC-SA 4.0 (https://creativecommons.org/licenses/by-nc$\mathrm{sa} / 4.0 /)$

La coagulación utilizando sulfato de aluminio ayudó a mejorar la calidad del agua. Datos de los parámetros calidad del agua antes y después de algunas de algunas de la prueba de jarras realizadas se muestran en la Tabla 1. Se observa cómo después de agregar el sulfato de aluminio y lograr la sedimentación, el pH, colory turbiedad del agua tratada disminuye, mientras que el tratamiento conlleva a un aumento de la conductividad eléctrica.

Tabla 1. Datos de la calidad del agua antes y después del tratamiento de coagulación durante la prueba de jarras.

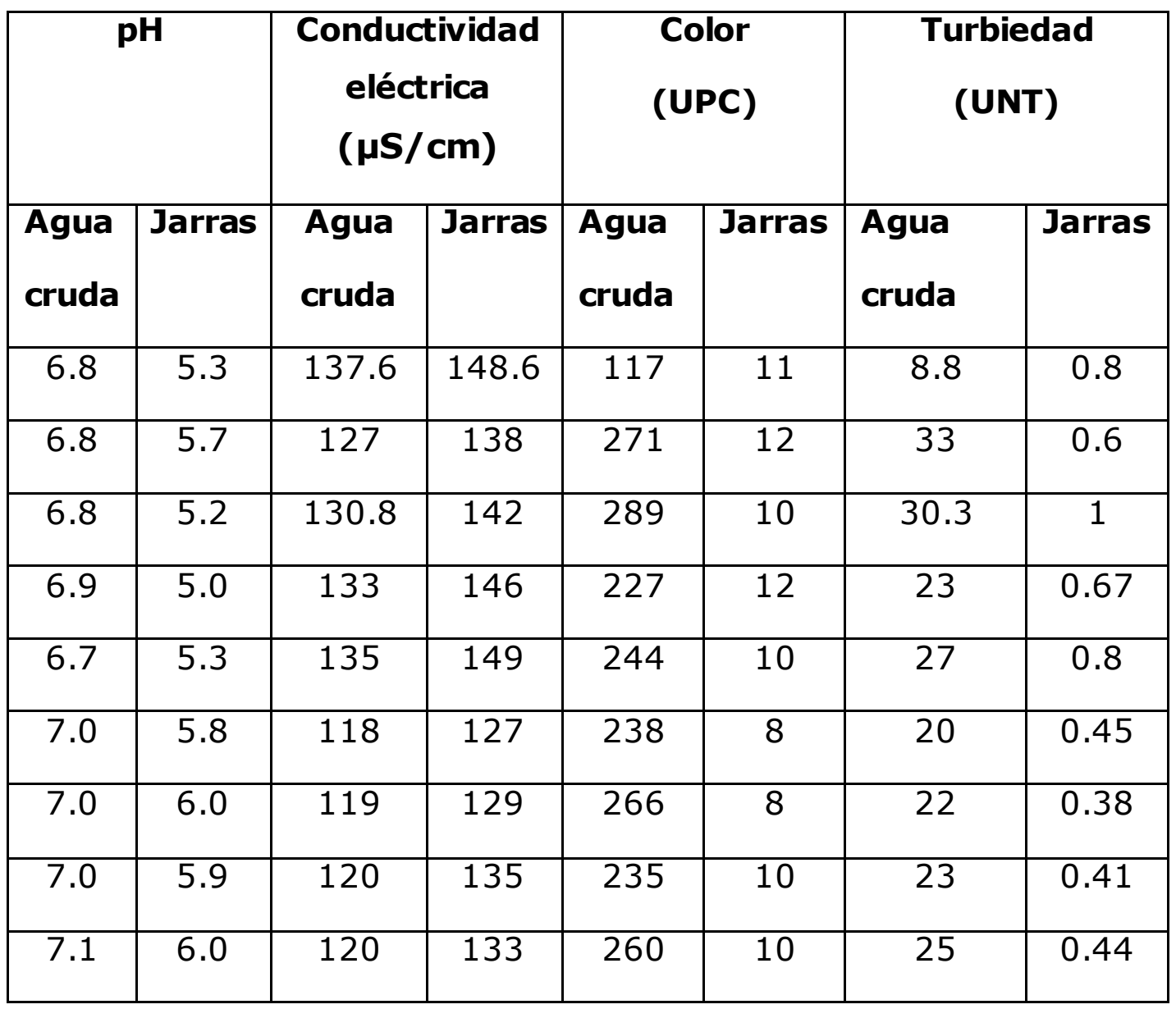




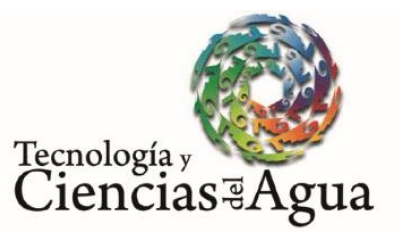

2020, Instituto Mexicano de Tecnología del Agua

Open Access bajo la licencia CC BY-NC-SA 4.0 (https://creativecommons.org/licenses/by-nc$\mathrm{sa} / 4.0 /$ )

\section{Asociación entre variables}

Los coeficientes de correlación de Pearson obtenidos entre pares de variables se exponen a través de la matriz de correlación en la Tabla 2. La asociación entre la dosis del coagulante con variables como pH, conductividad eléctrica, color, turbiedad del agua cruda y dosis del polímero se indica por los valores de correlación de $0.757,0.615,0.712,0.743$ y 0.404 , respectivamente. Este tipo de coeficientes de correlación no son muy altos, así que se puede considerar que no hay colinealidad entre variables y, por lo tanto, la dimensión de los datos experimentales se conserva. En conclusión, la dosis de coagulante se determina por $\mathrm{pH}$, turbiedad, conductividad eléctrica y color del agua cruda, así como por la dosis de polímero floculante. Estas observaciones apuntan a que se puede encontrar un modelo que prediga la dosis del coagulante a partir de $\mathrm{pH}$, conductividad eléctrica, turbiedad, color del agua cruda y dosis del floculante, y que todas estas variables son independientes con respecto a la dosis de coagulante. Dichos valores de correlación garantizan que la red neuronal converge en un conjunto de pesos sinápticos óptimos.

Tabla 2. Matriz de correlación para los datos experimentales. 


\begin{tabular}{|c|c|c|c|c|c|c|}
\hline \multicolumn{2}{|c|}{$\begin{array}{l}\text { Tecnología y } \\
\text { Ciencias } \approx \text { Agua }\end{array}$} & \multicolumn{5}{|c|}{$\begin{array}{l}\text { 2020, Instituto Mexicano de Tecnología del Agua } \\
\text { Open Access bajo la licencia CC BY-NC-SA } 4.0 \\
\text { (https://creativecommons.org/licenses/by-nc- } \\
\text { sa/4.0/) }\end{array}$} \\
\hline Parámetro & pH & $\begin{array}{l}\text { Conductividad } \\
\text { eléctrica } \\
(\mu \mathrm{S} / \mathrm{cm})\end{array}$ & $\begin{array}{l}\text { Color } \\
\text { (UPC) }\end{array}$ & $\begin{array}{c}\text { Turbiedad } \\
\text { (UNT) }\end{array}$ & $\begin{array}{l}\text { Dosis del } \\
\text { polímero } \\
(\mathrm{ppm})\end{array}$ & $\begin{array}{l}\text { Dosis del } \\
\text { coagulante } \\
\text { (ppm) }\end{array}$ \\
\hline $\mathrm{pH}$ & 0.979 & 0.639 & 0.697 & 0.754 & 0.373 & 0.757 \\
\hline $\begin{array}{c}\text { Conductividad } \\
\text { eléctrica }\end{array}$ & 0.639 & 0.971 & 0.833 & 0.804 & 0.781 & 0.615 \\
\hline Color & 0.697 & 0.833 & 0.972 & 0.928 & 0.660 & 0.712 \\
\hline Turbiedad & 0.754 & 0.804 & 0.928 & 0.973 & 0.580 & 0.743 \\
\hline $\begin{array}{l}\text { Dosis del } \\
\text { polímero }\end{array}$ & 0.373 & 0.781 & 0.044 & 0.580 & 0.984 & 0.404 \\
\hline $\begin{array}{c}\text { Dosis del } \\
\text { coagulante }\end{array}$ & 0.757 & 0.615 & 0.712 & 0.743 & 0.404 & 0.979 \\
\hline
\end{tabular}

\section{Validación del modelo}

Se utilizó un típico perceptrón de tres capas. La primera capa correspondió a la entrada, donde se introdujeron los 27 datos experimentales del entrenamiento en forma de vectores. Todos los valores fueron escalados entre 0 y 1 , sustrayendo el valor más bajo, y dividiéndolos por la diferencia entre máximo y mínimo. El valor máximo tuvo un valor de 1 ; el valormedio, 0.5; el valor mínimo, 0, como se observa en la Tabla 3. 
Tecnología y

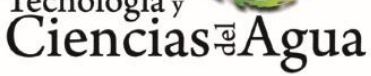

2020, Instituto Mexicano de Tecnología del Agua Open Access bajo la licencia CC BY-NC-SA 4.0 (https://creativecommons.org/licenses/by-nc$\mathrm{sa} / 4.0 /)$

Tabla 3. Valores mínimos, máximos y medio de los rangos para las variables independientes y la variable dependiente.

\begin{tabular}{|l|l|l|l|l|l|l|}
\hline & $\mathbf{p H}$ & $\begin{array}{l}\text { Turbiedad } \\
(\mathbf{U N T})\end{array}$ & $\begin{array}{l}\text { Color } \\
(\mathbf{U P C})\end{array}$ & $\begin{array}{l}\text { Conductividad } \\
\text { eléctrica } \\
(\boldsymbol{\mu S} / \mathbf{c m})\end{array}$ & $\begin{array}{l}\text { Dosis de } \\
\text { floculante } \\
\mathbf{( p p m )}\end{array}$ & $\begin{array}{l}\text { Dosis de } \\
\text { coagulante } \\
(\mathbf{p p m})\end{array}$ \\
\hline Valor mínimo & 6.7 & 7 & 117 & 118.5 & 0 & 35 \\
\hline Valor máximo & 7.3 & 33.1 & 289 & 202 & 0.7 & 59 \\
\hline Valor medio & 7 & 20.05 & 203 & 160.25 & 0.35 & 47 \\
\hline
\end{tabular}

Puesto que cada dato experimental escalado en forma de vector tiene cinco dimensiones correspondientes a $\mathrm{pH}$, conductividad eléctrica, turbiedad, color y dosis del floculante, la capa de entrada tiene cinco neuronas. Cada una de las cinco neuronas de las capa de entrada se conectan a cada una de las neuronas de la única capa oculta a través de cinco conexiones, y cada conexión tiene un peso sináptico. Las cinco componentes de los puntos experimentales escalados se multiplican por los cinco pesos simpáticos; luego se suman para transferir la información a una función sigmoidea.

La información procesada en cada una de las neuronas en la capa oculta se propaga a la capa de salida. Para enviar estos datos a la única neurona en la capa de salida, los datos que vienen de cada una de las neuronas en la capa oculta se multiplican por otro grupo de pesos sinápticos que caracterizan las conexiones desde cada una de las neuronas en la capa oculta con la única neurona en la capa de salida. Esta información se procesa en la única neurona, en la capa de salida, a través de una segunda función sigmoidea, donde se obtiene el valor predicho por el modelo.

Para encontrar el número óptimo de neuronas en la capa oculta, se 
Tecnología y

Ciencias $\approx$ Agua
2020, Instituto Mexicano de Tecnología del Agua Open Access bajo la licencia CC BY-NC-SA 4.0 (https://creativecommons.org/licenses/by-nc$\mathrm{sa} / 4.0 /)$

analizaron redes neuronales con $2,4,6,8,10$ y 12 neuronas en la capa oculta a través de validación cruzada. El programa Solver de Microsoft se usó para entrenarla red neuronal de tres capas. EVC se calculó para los modelos que tenían el mismo número de neuronas en la capa oculta, como se observa en la Tabla 4. El tipo de redes neuronales que tuvo el EVC más bajo fue aquel con 10 neuronas en la capa oculta. La Figura 2 muestra el efecto del número de neuronas en la capa oculta sobre el EVC. Se utilizó un perceptrón de tres capas, con 5, 10 y 1 neurona para la capa de entrada, oculta y de salida, respectivamente.

Tabla 4. ECP de los 162 modelos construidos y EVC para cada tipo de modelo con número definido de neuronas en la capa oculta.

\begin{tabular}{|c|c|c|c|c|c|c|}
\hline & \multicolumn{5}{|c|}{ Número de neuronas en la capa oculta } \\
\hline Modelos & $\mathbf{2}$ & $\mathbf{4}$ & $\mathbf{6}$ & $\mathbf{8}$ & $\mathbf{1 0}$ & $\mathbf{1 2}$ \\
\hline $\mathbf{1}$ & 0.9083 & 0.4072 & 0.3921 & 0.2208 & 0.0921 & 1.1578 \\
\hline $\mathbf{2}$ & 0.9162 & 0.3884 & 0.3809 & 0.2208 & 0.0070 & 1.1040 \\
\hline $\mathbf{3}$ & 0.0079 & 0.2956 & 0.2718 & 0.1470 & 0.0916 & 0.8743 \\
\hline $\mathbf{4}$ & 0.9101 & 0.4074 & 0.3921 & 0.2209 & 0.0921 & 1.1579 \\
\hline $\mathbf{5}$ & 0.9149 & 0.4075 & 0.3807 & 0.2210 & 0.0921 & 1.1578 \\
\hline $\mathbf{6}$ & 0.8748 & 0.4074 & 0.3920 & 0.2208 & 0.0921 & 1.1577 \\
\hline $\mathbf{7}$ & 0.8964 & 0.4075 & 0.3922 & 0.1933 & 0.0921 & 1.1579 \\
\hline $\mathbf{8}$ & 0.8993 & 0.4075 & 0.3923 & 0.2210 & 0.0886 & 1.1579 \\
\hline $\mathbf{9}$ & 0.8512 & 0.4013 & 0.3923 & 0.2202 & 0.0892 & 1.1579 \\
\hline
\end{tabular}


2020, Instituto Mexicano de Tecnología del Agua

Tecnología y

Ciencias $₫$ Agua
Open Access bajo la licencia CC BY-NC-SA 4.0 (https://creativecommons.org/licenses/by-nc$\mathrm{sa} / 4.0 /)$

\begin{tabular}{|c|c|c|c|c|c|c|}
\hline 10 & 0.9084 & 0.4075 & 0.3923 & 0.2206 & 0.0920 & 1.1579 \\
\hline 11 & 0.9018 & 0.4073 & 0.3922 & 0.2209 & 0.0921 & 1.1579 \\
\hline 12 & 0.9101 & 0.4075 & 0.3922 & 0.2209 & 0.0921 & 1.1579 \\
\hline 13 & 0.9101 & 0.4075 & 0.3922 & 0.2200 & 0.0921 & 1.1579 \\
\hline 14 & 0.9101 & 0.4075 & 0.3923 & 0.2197 & 0.0921 & 1.1579 \\
\hline 15 & 0.8340 & 0.4075 & 0.3923 & 0.2207 & 0.0921 & 1.1579 \\
\hline 16 & 0.8352 & 0.3542 & 0.3363 & 0.2206 & 0.0921 & 1.1553 \\
\hline 17 & 0.9101 & 0.3965 & 0.3776 & 0.2206 & 0.0921 & 1.1553 \\
\hline 18 & 0.9161 & 0.4054 & 0.3923 & 0.2203 & 0.0921 & 1.1329 \\
\hline 19 & 0.9105 & 0.4069 & 0.3922 & 0.2210 & 0.0921 & 1.1329 \\
\hline 20 & 0.9153 & 0.4060 & 0.3918 & 0.2089 & 0.0921 & 1.1329 \\
\hline 21 & 0.9101 & 0.3896 & 0.3738 & 0.1833 & 0.0921 & 1.1329 \\
\hline 22 & 0.9104 & 0.4069 & 0.3923 & 0.2184 & 0.0921 & 1.1329 \\
\hline 23 & 0.8340 & 0.4065 & 0.3893 & 0.2194 & 0,0921 & 1.1329 \\
\hline 24 & 0.9162 & 0.4031 & 0.3917 & 0.2210 & 0.0921 & 1.1552 \\
\hline 25 & 0.9101 & 0.3903 & 0.3811 & 0.2210 & 0.0921 & 1.1553 \\
\hline 26 & 0.8018 & 0.3972 & 0.3819 & 0.2210 & 0.0919 & 0.9398 \\
\hline 27 & 0.9101 & 0.3981 & 0.3866 & 0.2206 & 0.0921 & 1.1553 \\
\hline EVC & 0.8564 & 0.3934 & 0.3831 & 0.2140 & 0.0888 & 1.1255 \\
\hline
\end{tabular}


2020, Instituto Mexicano de Tecnología del Agua

Tecnología y

Ciencias $\stackrel{\Xi}{\approx}$ gua
Open Access bajo la licencia CC BY-NC-SA 4.0 (https://creativecommons.org/licenses/by-ncsa/4.0/)

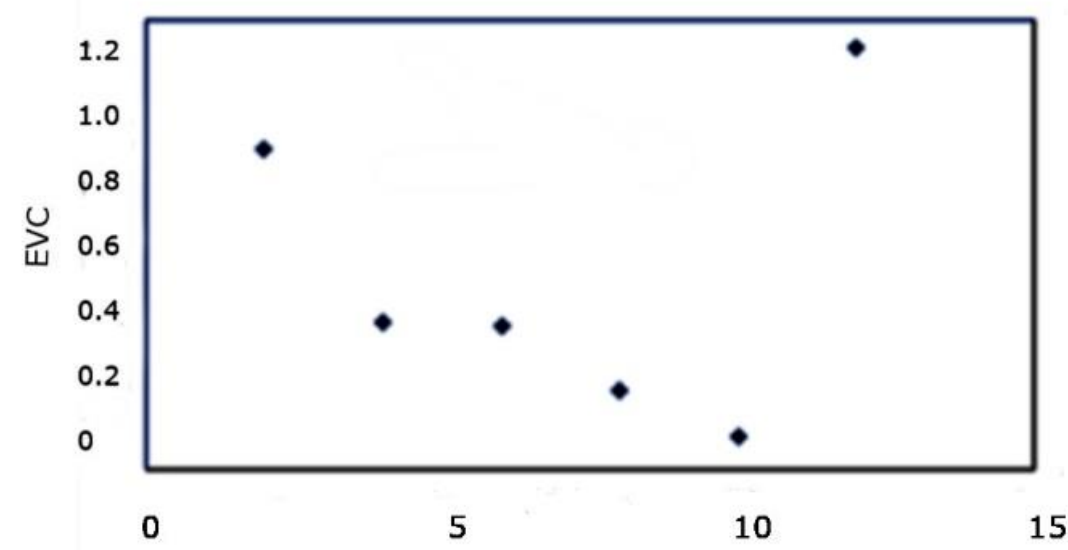

Número de neuronas en la capa oculta

Figura 2. Efecto del número de neuronas en la capa oculta sobre el EVC.

\section{Evaluación del modelo}

Los datos experimentales empleados para entrenar el modelo fueron 27 y 4 datos obtenidos, que se usaron para la evaluación del modelo. La Tabla 5 expone los valores predichos para los datos de entrenamiento y los utilizados para la evaluación del modelo. Los residuales para los datos de entrenamiento están entre -1.47 y 1.03 , y son residuales bajos, a causa de que los datos de entrenamiento se utilizaron para encontrar los parámetros del modelo. 
Tecnología y

Ciencias 웜ua
2020, Instituto Mexicano de Tecnología del Agua Open Access bajo la licencia CC BY-NC-SA 4.0 (https://creativecommons.org/licenses/by-ncsa/4.0/)

Tabla 5. Datos de entrenamiento y predicción para el modelo desarrollado.

\begin{tabular}{|c|c|c|c|c|c|c|c|c|}
\hline \multicolumn{9}{|c|}{ Datos de entrenamiento } \\
\hline & pH & $\begin{array}{c}\text { Cond. } \\
\text { eléctrica } \\
(\mu s / \mathrm{cm})\end{array}$ & $\begin{array}{l}\text { Color } \\
\text { (UPC) }\end{array}$ & $\begin{array}{l}\text { Turbiedad } \\
\text { (UNNT) }\end{array}$ & $\begin{array}{c}\text { Dosis del } \\
\text { floculante } \\
\text { (ppm) }\end{array}$ & $\begin{array}{c}\text { Dosis } \\
\text { coagulante } \\
\text { experimental } \\
\text { (ppm) }\end{array}$ & $\begin{array}{l}\text { Dosis } \\
\text { según } \\
\text { modelo }\end{array}$ & $\begin{array}{c}\text { Residual } \\
\qquad-y\end{array}$ \\
\hline 1 & 6.8 & 170.3 & 132 & 13 & 0 & 54 & 55.00 & -1.00 \\
\hline 2 & 6.9 & 162.7 & 252 & 25.7 & 0 & 45 & 45.24 & -0.24 \\
\hline 3 & 7.1 & 128.9 & 282 & 27.3 & 0 & 44 & 44.18 & -0.18 \\
\hline 4 & 6.9 & 136.9 & 217 & 20.5 & 0 & 48 & 49.08 & -1.08 \\
\hline 5 & 6.8 & 137.6 & 117 & 8.87 & 0 & 55 & 55.00 & 0.00 \\
\hline 6 & 6.8 & 130.8 & 289 & 30.3 & 0 & 40 & 39.76 & 0.24 \\
\hline 7 & 6.8 & 127.1 & 271 & 33.1 & 0 & 37 & 38.47 & -1.47 \\
\hline 8 & 6.7 & 135 & 244 & 27 & 0 & 41 & 40.13 & 0.87 \\
\hline 9 & 6.9 & 133 & 227 & 23 & 0 & 46 & 44.97 & 1.03 \\
\hline 10 & 7 & 118.5 & 238 & 20.3 & 0.6 & 45 & 44.98 & 0.02 \\
\hline 11 & 7 & 119. 5 & 266 & 22.7 & 0.6 & 45 & 44.85 & 0.15 \\
\hline 12 & 7 & 120.5 & 235 & 23.3 & 0.6 & 45 & 44.92 & 0.08 \\
\hline 13 & 7.1 & 120 & 260 & 25.3 & 0.7 & 50 & 50.18 & -0.18 \\
\hline 14 & 7 & 174 & 130 & 7.16 & 0 & 45 & 45.12 & -0.12 \\
\hline 15 & 7.1 & 174.8 & 177 & 13.8 & 0 & 45 & 45.21 & -0.21 \\
\hline 16 & 7 & 202 & 204 & 15.1 & 0 & 50 & 49.83 & 0.17 \\
\hline 17 & 7.1 & 189 & 135 & 9.95 & 0 & 50 & 50.51 & -0.51 \\
\hline
\end{tabular}


2020, Instituto Mexicano de Tecnología del Agua

Tecnología y

Ciencias $₫$ Agua
Open Access bajo la licencia CC BY-NC-SA 4.0 (https://creativecommons.org/licenses/by-nc$\mathrm{sa} / 4.0 /)$

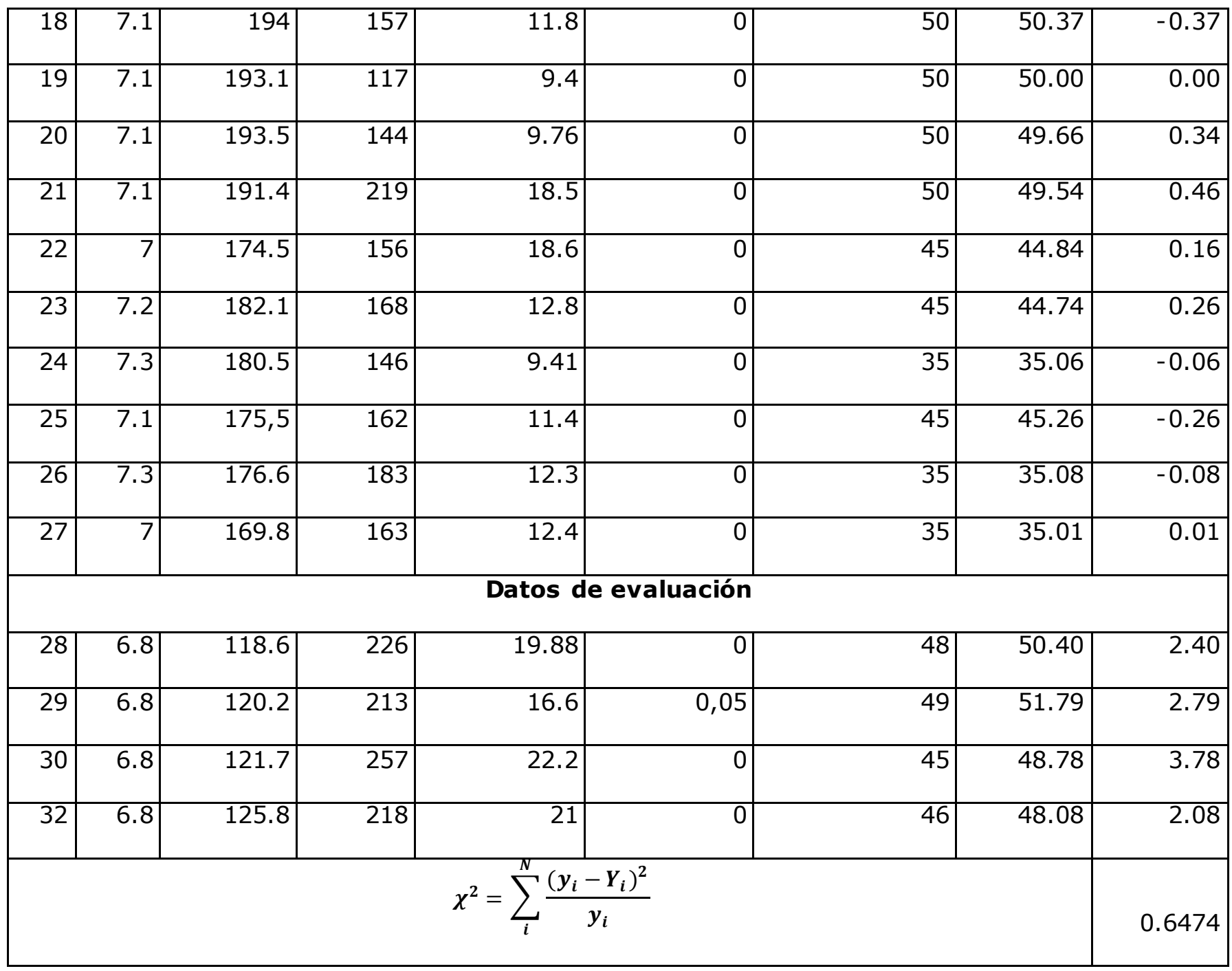

Para evaluar el modelo es importante usar un conjunto de datos que no pertenezcan a los datos experimentales de entrenamiento, así que se emplea un conjunto de cuatro. El valor chi cuadrada es de 0.6474 para los datos experimentales de evaluación; es menor que el valor chi cuadrado crítico de 6.21 a 90\% de confianza, con tres grados de libertad. Por lo tanto, la hipótesis nula se acepta, lo que conlleva a concluir que los valores predichos por el modelo para datos experimentales de evaluación son consistentes con los observados. 
Tecnología y

Ciencias Agua
2020, Instituto Mexicano de Tecnología del Agua Open Access bajo la licencia CC BY-NC-SA 4.0 (https://creativecommons.org/licenses/by-nc$\mathrm{sa} / 4.0 /)$

\section{Discusión}

Las relaciones entre turbiedad y dosis de coagulante con respecto al $\mathrm{pH}$, color, conductividad eléctrica y dosis de polímero, según el modelo matemático desarrollado, se exponen en los gráficos de contorno en la Figura 3 y Figura 4.
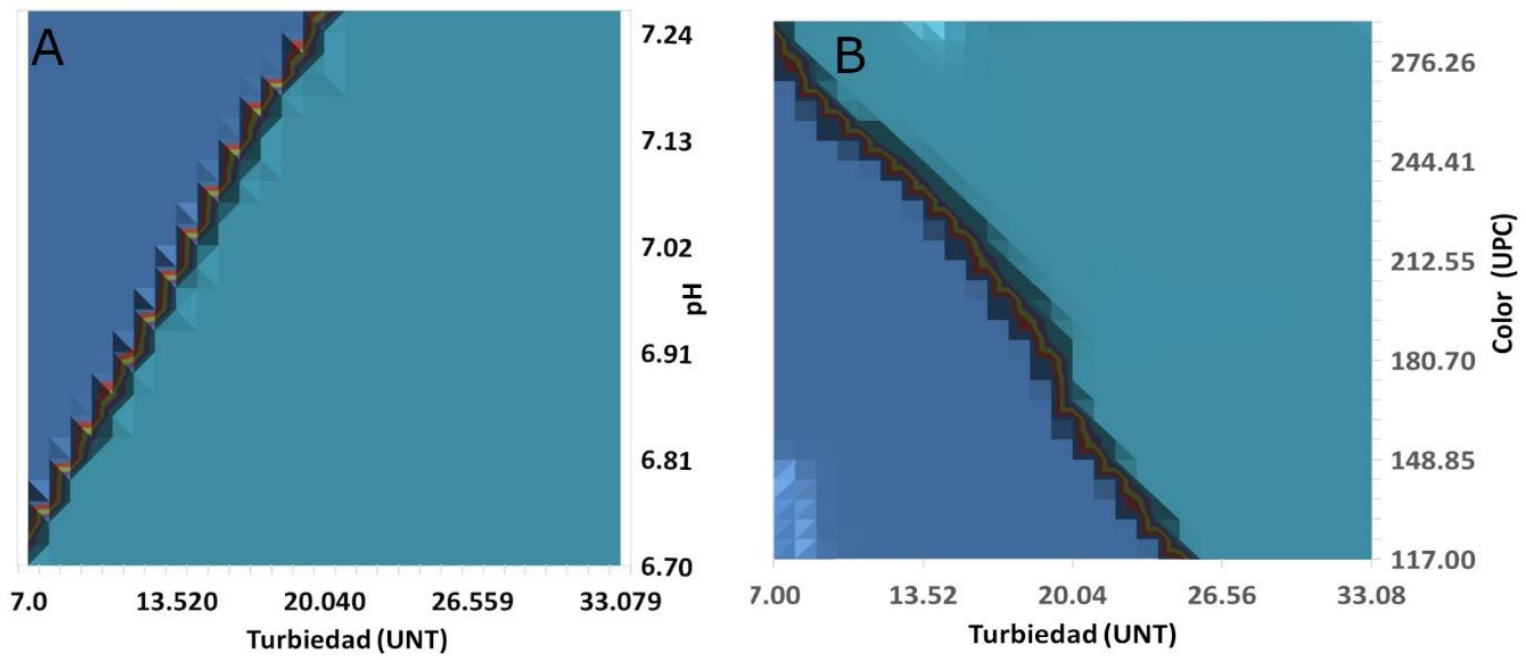

- 35-39.5

54.2-59 ppm

Figura 3. Efecto de la turbiedad, $\mathrm{pH}$ y color en la dosis de coagulante. En el gráfico A se observa el efecto de la turbiedad y el $\mathrm{pH}$, mientras que en el gráfico B se observa el efecto de la turbiedad y el color. En la barra inferior 


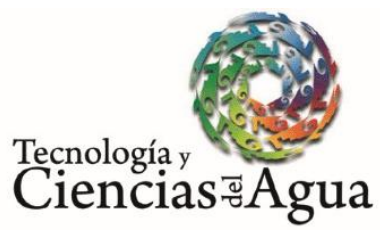

2020, Instituto Mexicano de Tecnología del Agua Open Access bajo la licencia CC BY-NC-SA 4.0 (https://creativecommons.org/licenses/by-ncsa/4.0/)

se indican los rangos de los valores de dosis de coagulante predichos por el modelo, indicados por diferente color.
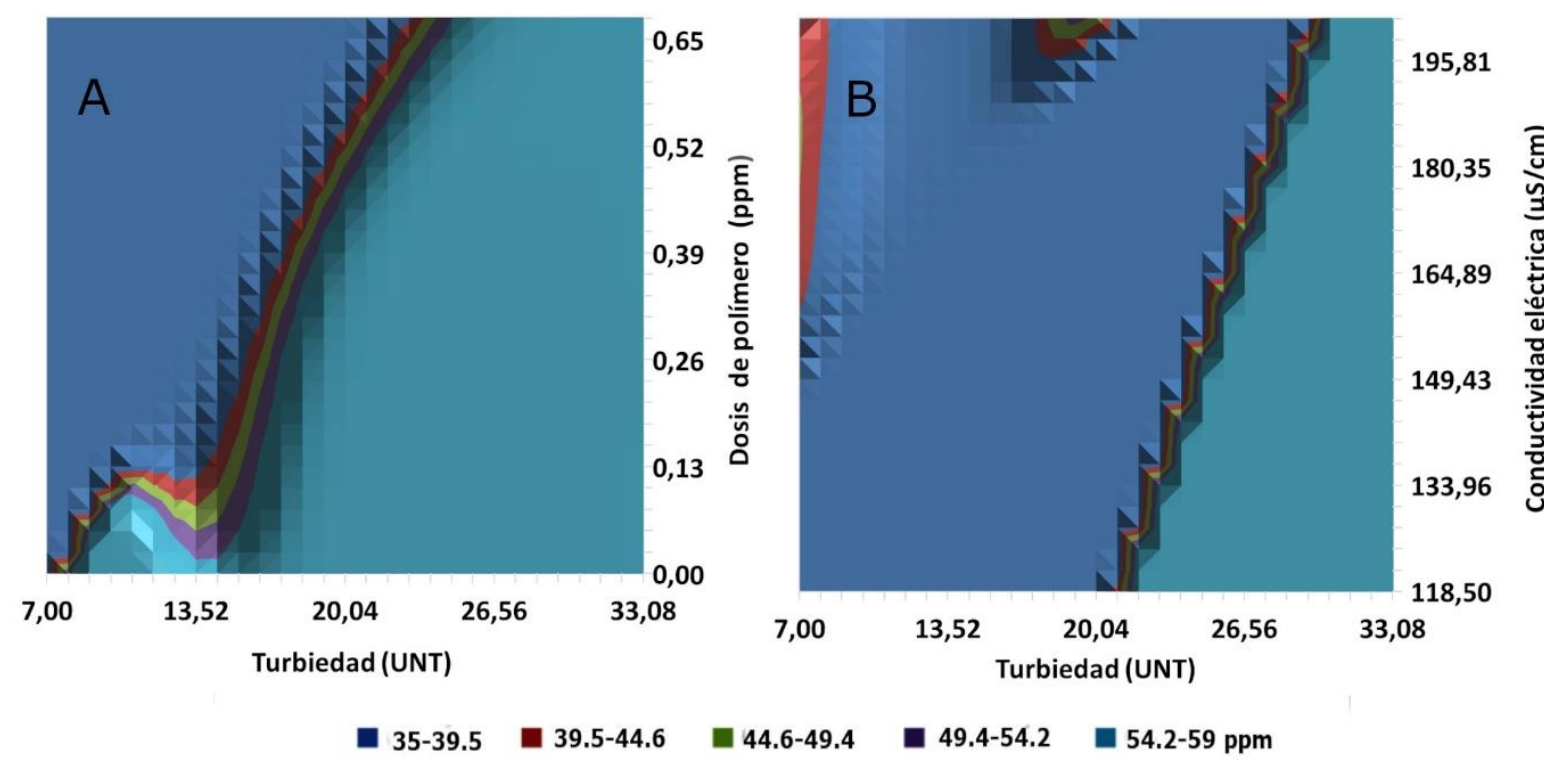

Figura 4. Efecto de la turbiedad, conductividad eléctrica y dosis de polímero en la dosis de coagulante. En el gráfico A advierte el efecto de la turbiedad y la conductividad eléctrica, mientras que el gráfico B indica el efecto de la turbiedad y la dosis de polímero. En la barra inferior se presentan rangos de los valores de dosis de coagulante predichos por los modelo, indicados por diferente color.

La Figura 3(A) expone un gráfico de contorno, donde el eje $X$ corresponde a turbiedad; el eje $Y$, a pH; y el eje $Z$, a la dosis de coagulante. Se asumieron para el color y la conductividad eléctrica valores mínimos y 


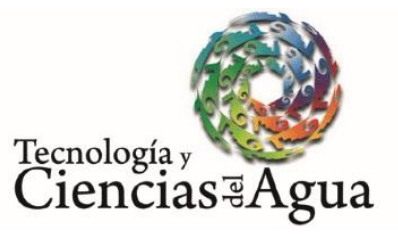

2020, Instituto Mexicano de Tecnología del Agua Open Access bajo la licencia CC BY-NC-SA 4.0 (https://creativecommons.org/licenses/by-nc$\mathrm{sa} / 4.0 /)$

constantes de 117 UPC y $118.5 \mu \mathrm{S} / \mathrm{cm}$, respectivamente. Además, la dosis del polímero se mantuvo en cero.

La Figura 3(A) muestra dos zonas: una caracterizada por valores de turbiedad mayores de 20 UNT, donde la dosis de coagulante predicha por el modelo está entre 54 y 59 ppm, y es independiente del pH. En la región de valores de turbiedad por debajo de 20 UNT, el pH empieza a tener efecto sobre la dosis de coagulante. Se observa por debajo de 20 UNT que, a medida que se disminuyen los valores de $\mathrm{pH}$ y se incrementa la turbiedad, la dosis de coagulante continúa manteniéndose en valores entre 54 y 59 ppm, mientras que un aumento en $\mathrm{pH}$ y una disminución en turbiedad logran disminuir la dosis de coagulante a valores entre 35 y 39 ppm.

La Figura 3(B) muestra en el eje $X$ la turbiedad; en el eje $Y$, al color; $y$ en el eje $Z$, la dosis de coagulante. Se asumieron valores de $\mathrm{pH}$ y conductividad eléctrica de 7.3 y $118.5 \mu \mathrm{S} / \mathrm{cm}$, respectivamente. El valor de la dosis de polímero se mantuvo en cero.

De nuevo se identifica una zona de mayor turbiedad por encima de 26 UNT, caracterizada por valores de dosis de coagulante entre 54 y 59 ppm, independientes del color. Cuando la turbiedad está por debajo de 26 UNT, la dosis de coagulante es afectada por el color, pues cuando el valor del color empieza a aumentar y la turbiedad disminuye, la dosis del coagulante continúa manteniéndose entre 54 y 59 ppm. A la vez, en la misma región por debajo de 25 UNT, la disminución de color y aumento de turbiedad conduce a que la dosis de coagulante baje a valores entre 35 y 39 ppm de dosis de coagulante.

Si se compara el efecto del $\mathrm{pH}$ y del color, se puede observar que un aumento de $\mathrm{pH}$ y una merma de color favorecen la reducción de la dosis de 


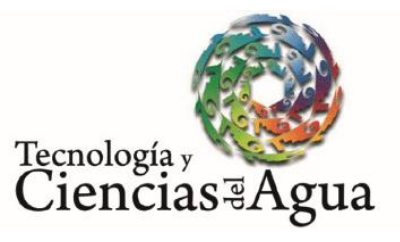

2020, Instituto Mexicano de Tecnología del Agua Open Access bajo la licencia CC BY-NC-SA 4.0 (https://creativecommons.org/licenses/by-ncsa/4.0/)

coagulante si la turbiedad está por debajo de 26 UNT; mientras que por encima de 26 UNT, la dosis de coagulante es independiente del colory del $\mathrm{pH}$.

La Figura 4(A) muestra en el eje $X$ la turbiedad; en el eje $Y$, la conductividad eléctrica; y en el eje $Z$, la dosis de coagulante. Se asumió para el $\mathrm{pH}$ y el color valores de 7.3 y 117 UPC, respectivamente. La dosis del polímero se mantuvo en cero.

Se observó una zona para valores de turbiedad por debajo de 20 UNT, donde la dosis de coagulante predicha por el modelo está en valores entre 35 y 39 ppm, y es independiente de la conductividad eléctrica.

La conductividad eléctrica empieza a tener efecto sobre la dosis de coagulante en valores de turbiedad entre 20 y 30 UNT. En este rango de turbiedades, donde hay mayor conductividad, se mantiene la dosis de coagulante entre 35 y 39 ppm, y una menor conductividad podría aumentar la dosis de coagulante hasta valores entre 54 y 59 ppm. Después de 30 UNT, la dosis de coagulante se hace independiente de la conductividad, para continuar teniendo valores entre 54 y 59 ppm.

El efecto de la dosis de polímero evidencia que a mayores valores de 25 UNT de turbiedad, la dosis de coagulante predicha entre 54 y 59 ppm es independiente de la dosis de polímero; mientras que a valores de turbiedad menores de 25 UTN, un aumento en la dosis de polímero ayuda a reducir la dosis de coagulante a valores entre 35 y 39 ppm. Este crecimiento en la dosis de polímero es posible sólo hasta $1 \mathrm{ppm}$ por motivos de seguridad, debido a la toxicidad de la poliacrilamida aniónica. Igualmente se observa que no usar polímero sólo contribuiría al incremento de la dosis de coagulante. 


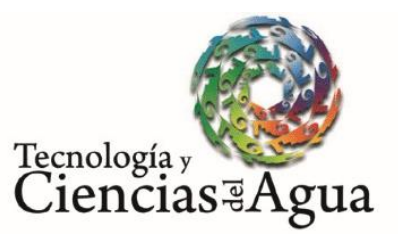

2020, Instituto Mexicano de Tecnología del Agua Open Access bajo la licencia CC BY-NC-SA 4.0 (https://creativecommons.org/licenses/by-nc$\mathrm{sa} / 4.0 /)$

\section{Conclusiones}

Un modelo de red neuronal basado en un perceptrón de tres capas permitió predecir la dosis de sulfato de aluminio usado como coagulante, a partir de variables independientes, como $\mathrm{pH}$, conductividad, color, turbiedad del agua cruda y dosis de polímero. La evaluación del modelo con base en una prueba de chi cuadrada arrojó resultados en los que el modelo es eficiente en un $90 \%$ de nivel de confianza. Los valores mayores de $\mathrm{pH}$, conductividad eléctrica, floculante de hasta $1 \mathrm{ppm}$ y menores valores de color permiten mantener la dosis de coagulante entre 35 y 39 ppm cuando la turbiedad no es relativamente alta. Después de un valor límite de turbiedad de alrededor de 26 ppm, ni el pH, conductividad, color y uso de floculantes favorecen una reducción en la dosis de coagulante y eleva hasta valores entre 54 y 59 ppm.

\section{Agradecimientos}

Los autores desean agradecer a GENSA S. A. E. S. P., Planta Termopaipa, Colombia, por el todo el apoyo durante los experimentos de prueba de jarras.

\section{Referencias}


Tecnología y

Ciencias $₫$ Agua
2020, Instituto Mexicano de Tecnología del Agua Open Access bajo la licencia CC BY-NC-SA 4.0 (https://creativecommons.org/licenses/by-nc$\mathrm{sa} / 4.0 /)$

Altowayti, W. A., Algaifi, H. A., Bakar, S. A., \& Shahir, S. (2019). The adsorptive removal of As (III) using biomass of arsenic resistan Bacillus thuringiensis strain WS3: Characteristics and modelling studies. Ecotoxicology and Environmental Safety, 172, 176-185.

Anders, U., \& Korn, O. (1999). Model selection in neural networks. Neural Networks, 12, 309-323.

Bhatnagar, A., \& Minocha, A. K. (2006). Conventional and nonconventional adsorbents for removal of pollutants from water - A review. Indian Journal of Chemical Technology, 13(3), 203-217. Recuperado de https://doi.org/10.3354/Ab00330

Hadzima-Nyarko, M., Nyarko, E. K., \& Ademovic, N. (2019). Modelling the influence of waste rubber on compressive strength of concrete by artificial neural networks. Materials, 12(4), 561.

Jayaweera, C., \& Azis, N. (2018). Reliability of principal component analysis and Pearson correlation coefficient, for application in artificial neural network model development, for water treatment plants. IOP Conference Series: Materials Science and Engineering, Kuala Lampur, Malasya.

Kennedy, M., Gandomi, A., \& Miller, C. (2015). Coagulation modeling using artificial neural networks to predict both turbidity and DOMPARAFAC component removal. Journal of Environmental Chemical Engineering, 3(4), 2829-2838.

Machado-Infante, J., Ramírez-Caballero, G., \& Barajas-Meneses, M. J. (2016). Study of the adsorption capacity of Fe(II) dissolved in water by using a mineral rich in Manganese Dioxide ( $\mathrm{MnO} 2$ ) from Colombia. DYNA, 83(196), 223-228. Recuperado de 
2020, Instituto Mexicano de Tecnología del Agua

Tecnología y

Ciencias $₫$ Agua
Open Access bajo la licencia CC BY-NC-SA 4.0 (https://creativecommons.org/licenses/by-nc$\mathrm{sa} / 4.0 /$ )

https://doi.org/10.15446/dyna.v83n196.53566

Mason, C., \& Perreault, W. (1991). Collinearity, power, and interpretation of multiple regression analysis. Journal of Marketing Research, 3, 268-280.

Michael, K., Trinogga, J., \& Cebrian-Piqueras, M. (2019). Trait correlation network analysis identifies biomass allocation traits and stem specific length as hub traits in herbaceous perennial plants. Journal of Ecology. Recuperado de https://doi.org/10.1111/1365-2745.13066

Mojiri, A., Kazeroon, R. A., \& Gholami, A. (2019). Cross-linked magnetic chitosan/activated biochar for removal of emerging micropollutants from water: Optimization by the artificial neural network. Water, $11(3), 551$.

Olusola, O. E., Olurotimi, A. D., Adekilekun, J. L., \& Adetayo, A. J. (2019). Kinetics and neuro-fuzzy soft computing modelling of river turbid water coag-flocculation using mango (Mangifera indica) kernel coagulant. Chemical Engineering Communications, 206(2), 254-267.

Rao, S. S. (2009). Engineering optimization: Theory and practice ( $5^{\text {th }}$ ed.). Hoboken, USA: Wiley. 